\title{
Mechanisms of formation of solar pores and sunspots
}

\author{
Irina N. Kitiashvili \\ W.W. Hansen Experimental Laboratory and Center for Turbulence Research, \\ Stanford University, Stanford 94305, USA \\ email: irinasun@stanford.edu \\ Dept. of Astronomy, Kazan University \\ 420008, Kazan, Russia
}

\begin{abstract}
Spontaneous formation of self-organized magnetic structures, such as sunspots and pores, is one of intriguing and oldest problems, which represents a complicated interaction of convection and magnetic fields on different scales. Observations of sunspots and pores formation reveal a fast process of accumulation of emerging magnetic field into stable long-living magnetic structures. However, the physical mechanisms of the flux accumulation into the compact magnetic structures with high field strength and their stability are not clear. Development of observational capabilities, theory, and realistic-type MHD numerical simulations open a new level of our understanding of the turbulent processes of the magnetic field accumulation. I discuss the recent progress in observations and radiative MHD simulations that provide important clues for possible mechanisms of formation and stability of sunspots and pores, and their links to the dynamo process.
\end{abstract}

Keywords. Sun: magnetic fields, sunspots; methods: numerical

\section{Introduction}

Historically, the Sun was a key figure in many ancient cultures. It is unknown when sunspots are first detected, and usually this discovering is attributed to a pupil of Aristotle, in Theophrastus of Athens, in the middle of fourth century B.C. First systematic observations of sunspots started in China, Japan and Korea (see for review Bray \& Loughhead 1964; Solanki 2003). With the beginning of the telescopic era in the 17th century, ground and the space observations in the 20th century our knowledge of various aspects of the sunspots structure and dynamics has substantially expanded. However, still many important questions need to be understood. Periodical variations of the sunspot number indicate that the sunspot formation is connected to the global dynamo processes in the Sun, which are poorly understood. On the other hand, apparent self-organization of small-scale magnetic patches into the large-scale compact structures during the magnetic flux emergence shows the importance of the local dynamics of the turbulent convection.

Recent development of ground and space instrumentation, for high-spatial and spectral resolution for spectro-polarimetric data analysis technique make it possible to obtain a detail picture of flow and magnetic field dynamics in different wavelengths on the solar surface and atmosphere layers. Appearance of a new observational field of local helioseismology gives us unique information about the solar subsurface dynamics. Initiated by observed effects, theoretical models try to reconstruct the physical picture of these processes and have been able to predict new phenomena, discovered later in both observations and numerical simulations. Very recently, new computational capabilities make it possible to check old ideas and models, and to solve some fundamental long-standing problems. One of them is the understanding of the processes, which initiate the magnetic 


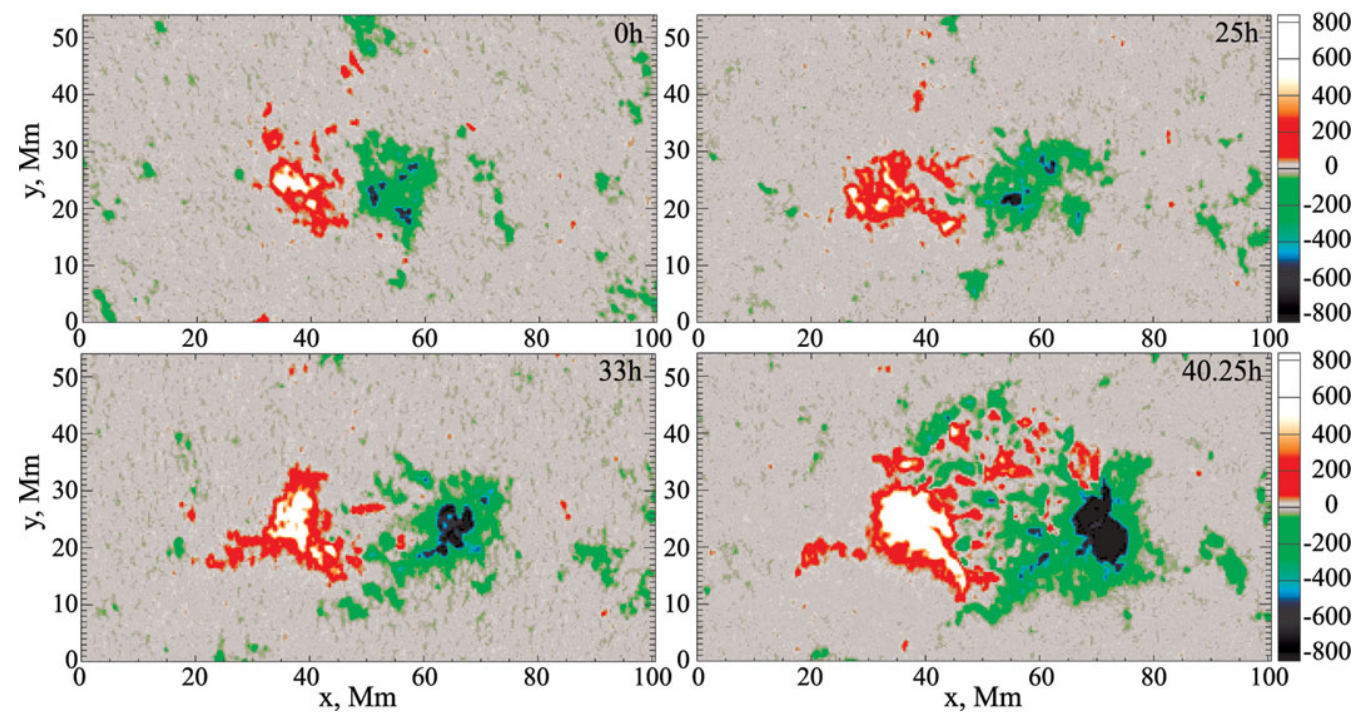

Figure 1. Evolution of AR11060 (April 4, 2010) observed by HMI/SDO shows emergence of magnetic flux and formation two compact sunspots.

field self-organization into compact stable structures and lead to formation of sunspots and pores. In this paper I will briefly discuss the current status of the problem, and start from a short overview of some observational facts, describe after this some theories of sunspots structure and dynamics, and finally discuss the recent progress and current problems of numerical MHD modeling of the solar magnetic structures.

\section{Observations of formation of magnetic structures}

Sunspots and pores represent compact concentrations of magnetic field on the surface. These structures are formed soon after emergence of magnetic flux generated by dynamo in the deep interior, properties of which are known from such observational facts as existence active longitudes, 22-year periodic magnetic cycles of activity, organization of magnetic structures in the form of bipolar active regions with leading sunspots having the same polarity in one heliosphere and the opposite polarity in the other heliosphere (Hale's polarity law) $\dagger$. Usually, the magnetic structures of the leading polarity are more compact than the following polarity structures, and form stable and long-living sunspots. The following polarity is often more diffusive and observed in the form of plages. There is no doubt that the overall bipolar structure of sunspot regions controlled by the largescale predominantly toroidal magnetic field generated by dynamo below the solar surface. However, why the magnetic field becomes concentrated in compact and stable sunspots remain a puzzle, therefore the first step to understanding of process formation sunspots and pores (observed in while light as smaller dark features) is to overview their observed properties.

\subsection{Flux emergence and formation of magnetic structures}

Emergence of the magnetic flux on the solar surface first causes disturbances of granulation, than forms a pore initially of the size of a single granule, which grows in time and

$\dagger$ This rule has only few violations in the history magnetic field observations. However, during the previous solar cycle 23, such violations of the sunspot 'polarity' rule showed up more often (Stenflo \& Kosovichev 2012). 

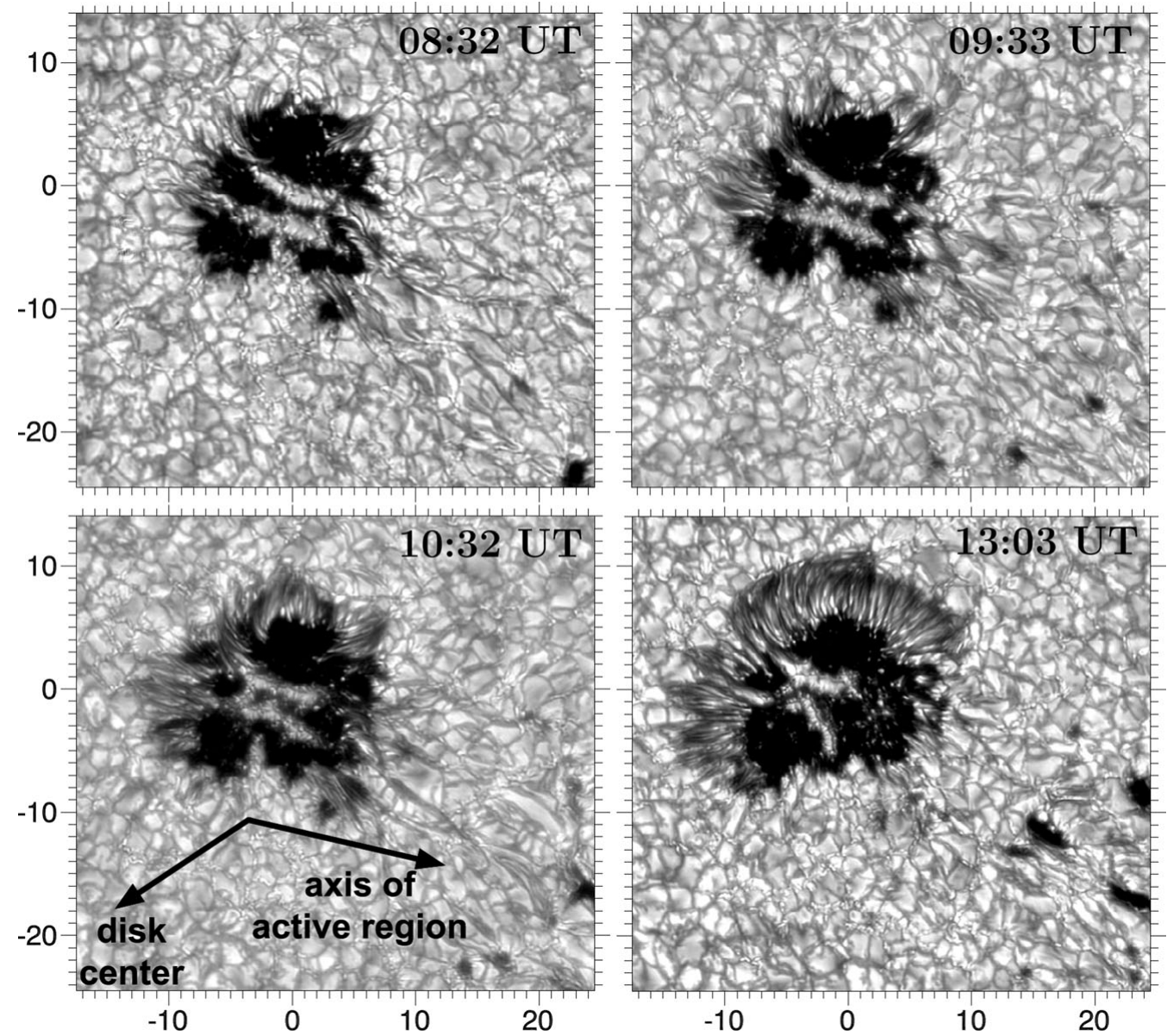

Figure 2. Observation of the penumbra formation for the sunspot NOAA 11024 (4 July, 2009) with the German Vacuum Tower Telescope (Schlichenmaier et al. 2010).

becomes darker (Loughhead \& Bray 1961). During the formation of an active region, the flux emergence may occur recurrently and often in several locations. Appearance of the flux on the surface is usually observed as two groups of small magnetic elements of the opposite polarity (e.g., Zirin 1972; Strous \& Zwaan 1999). The same polarity elements quickly move forward each other with a speed of about $\sim 0.5-1 \mathrm{~km} / \mathrm{s}$ (Wang \& Zirin 1992), merge and form compact opposite-polarity magnetic structures (Fig. 1). Often young sunspots show a strong rotation around their axis, which that slows down in time. The process of the sunspot formation on the solar surface from emerging magnetic flux takes usually from few hour to several days. Also, magnetic flux can continue emerge during the development of the sunspot active region. The 'new' flux emergence is usually detected near the leading and following edges (Zirin 1974). This flux may result in a growth of the initial sunspot structures, or complex magnetic configurations when an emerging flux tube has opposite polarity in the vicinity of a sunspot. Sometimes, this leads to formation of so-called 'delta-type' sunspots, when two 'umbras' of the opposite polarity appear close to each other within a common penumbra. Such spots are a primary source of powerful solar flares. 


\subsection{Surface properties of pores and sunspot}

Pores have a relatively simple structure and observed as compact small dark regions on the solar surface. the size of pores varies from subgranular ('micropores') to granular. A typical size of pores is $1-5 \mathrm{Mm}$ (Loughhead \& Bray 1961). Magnetic field in the photospheric layers of pores can reach up to $1-2 \mathrm{kG}$ and is predominantly vertical. Some pores show strong inhomogeneity with significant variations of the magnetic field inclination, $40-80^{\circ}$, and strength, $600-1700 \mathrm{G}$ (Sutterlin et al. 1996). The intensity deficit is $0.1-0.7 I_{p h}(\tau=1)$ and depends on the field strength (Zirin \& Wang 1992; Sobotka 2003). Detailed observations reveal small-scale granulation - 'umbral dots' inside pores (e.g., Sobotka et al. 1999, Hirzberger 2003 and references therein), which previously were identified in the sunspot umbra. The surrounding pore region is characterized by downflows that accelerate with depth in the lower photosphere layers, and also by converging inflows with a speed of about $0.5 \mathrm{~km} / \mathrm{s}$, in contrast to very weak flow motions inside a pore (Wang \& Zirin 1992; Keil et al. 1999; Roudier et al. 2002; Sankarasubramanian \& Rimmele 2003). Similar to the sunspot umbra, inside pores bright 'bridge' structures are observed, with significantly weaker magnetic field (e.g., Sobotka et al. 1999).

Nevertheless, despite the apparent simplicity of pores and their dynamics, the processes of pore transformation into sunspots and formation of the penumbra are still not understood. These processes was investigated by many authors (e.g., Bray \& Loughhead 1964; Sobotka et al. 1999), but only recently we are able to start high-resolution observations (Fig. 2, Schlichenmaier et al. 2010). These observations showed the initialization of the penumbra formation near the bright bridges, and subsequently, a growth of the penumbra along the whole 'pore' boundary. It is interesting that during the development of the penumbra, the strength of the magnetic field in the central 'umbral' part was kept stable, about $1.5 \mathrm{kG}$ (Rezaei et al. 2012).

It is well known, that not every pore develops into a sunspot, and the question is what is difference between these pores? Is this difference is caused by additional emerging flux or/and is this due to some specific properties of the pores? Effects of a new emerging flux on initialization of the penumbra formation may be significant because the new flux can change the magnetic field topology around a pore. The detection of moving magnetic features around some pores, which were previously observed only around the sunspot penumbra, gives an important dynamical link to a preexisting, but not yet formed penumbral magnetic topology, and can be a precursor of development of filamentary substructures (Zuccarello et al. 2009; Sainz Dalda et al. 2012).

Sunspots are some of the most prominent features on the solar surface, which can have very different sizes and levels of complexity. To account for the variety of the active region sizes and topological properties, the Zurich classification was developed (Waldmeier 1947; Kiepenheuer, 1953; McIntosh 1990). Nevertheless, despite the complexity two basic regions in sunspots can be identified: the umbra and penumbra.

The umbra is usually a compact area with very strong, up to $3 \mathrm{kG}$, mostly vertical magnetic field. High-resolution observations from the Swedish Solar Telescope, Hinode, Solar Dynamics Observatory (HMI instrument) and the BBSO New Solar Telescope have revealed the sunspot structure in great detail. The sunspot umbra contains numerous relatively bright 'umbral dots', which are about $\sim 300 \mathrm{~km}$ in diameter and have life-time of about 15 min. They are usually similar or smaller than these detected in pores. The umbral dots are characterized by slow upflow with a speed of about $0.1-1 \mathrm{~km} / \mathrm{s}$, weaker magnetic field (about $500 \mathrm{G}$ ) with about $10^{\circ}$ inclination to vertical. They have higher contrast and stronger field inclination, about $30^{\circ}$, near the umbra-penumbra boundary (e.g., Rimmele 2004; Socas-Navarro et al. 2004; Watanabe et al. 2009). The high-resolution 
observations also allowed to detect some fine substructure of the umbral dots (Rimmele 2004; Sobotka \& Puschmann 2009; Ortiz et al. 2010).

Dynamical and physical conditions of the penumbra are substantially differ from the umbral region, and represent a complicated system of the dark and bright filaments, 150 - $250 \mathrm{~km}$ wide, with almost horizontal magnetic field varying from $0.6-1 \mathrm{kG}$ at the sunspot edge to $\sim 2 \mathrm{kG}$ near the umbra. The dark penumbral filaments are associated with downflow regions and correspond to strong concentrations of almost horizontal magnetic field. In the bright filaments, high-resolution observations reveal a complicated dynamics such as their twisting. The observations also show dark cores in the bright penumbral filaments that moves inward into umbra region with speed about $1 \mathrm{~km} / \mathrm{s}$ (Severnyi 1959, Scharmer et al. 2002; Ichimoto et al. 2007). The dark cores of the bright penumbral filaments are often associated with radial outflows with a typical speed of 2 $4 \mathrm{~km} / \mathrm{s}$, known as the Evershed effect (e.g, Evershed 1909; Shine et al. 1994; Westendorp Plaza et al. 1997; Scharmer et al. 2002; Langhans et al. 2005; Bellot Rubio et al. 2005; Márquez et al. 2006; Rimmele \& Marino 2006; Rimmele 2008). In addition, quasi-periodic (with period $8-15 \mathrm{~min}$ ) high-speed, up to $7 \mathrm{~km} / \mathrm{s}$ outflows are observed in penumbra. Because of a similarity with the Evershed effect these flows are often called 'Evershed clouds'. They have a spatial scale of about $1 \mathrm{Mm}$, and can be separated in radius by 2 $3 \mathrm{Mm}$ (e.g., Shine et al. 1994; Rouppe van der Voort 2003, Cabrera Solana et al. 2008).

Despite that the magnetic field in the sunspot penumbra is almost horizontal, local topological properties of the field can vary; for instance in the bright filaments of penumbra the field lines are more vertical than in the dark filaments, representing the so-called 'uncombed' model (Solanki \& Montavon 1993; Martínez Pillet 2000; Sánchez Almeida 2005). Also, the local dynamic includes moving outward bipolar magnetic patches, which appear in an inner part of the penumbra (Sainz Dalda \& Bellot Rubio 2008), and can

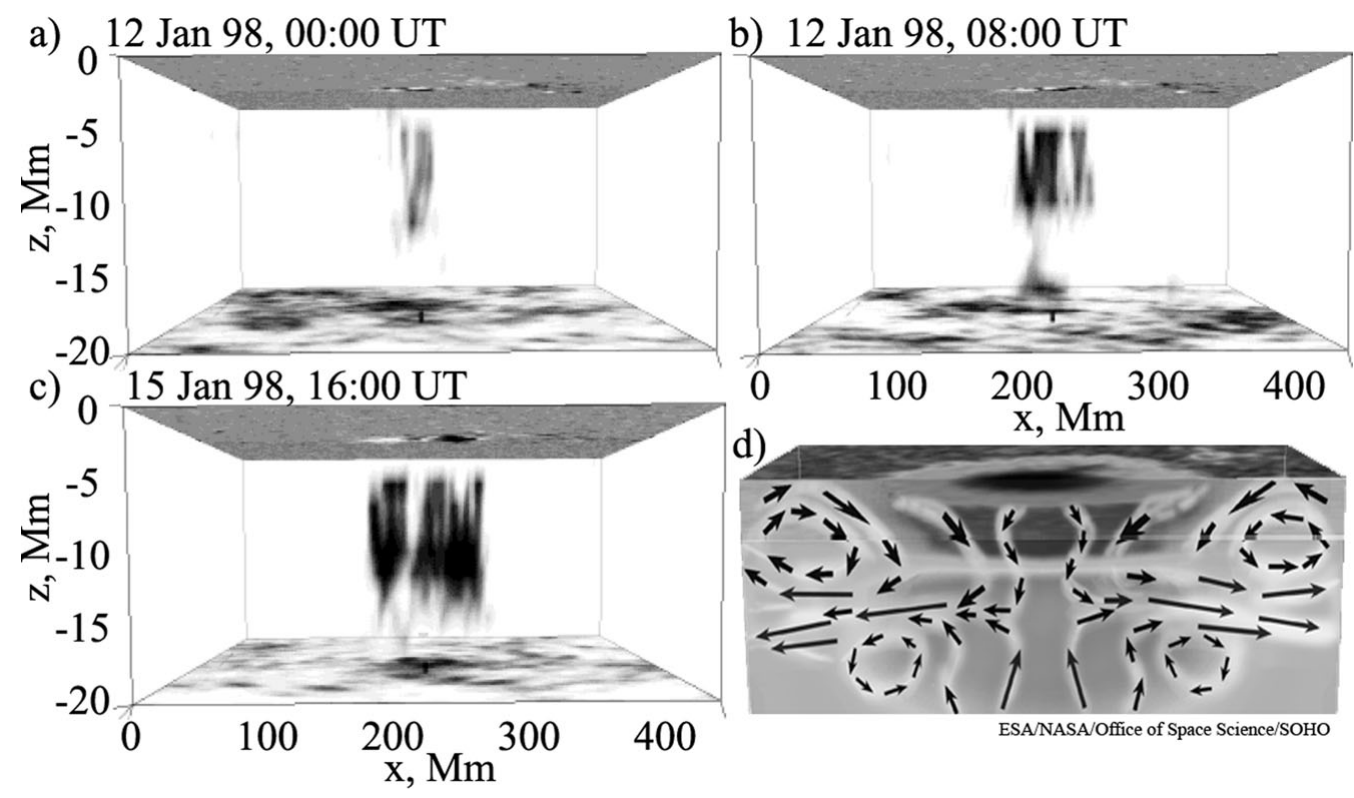

Figure 3. Active region subsurface dynamics obtained by time-distance helioseismology. Panels $a-c$ ) show the sound-speed perturbations range (color-scale is inverted) of emergence of active region NOAA 8131, the top planes are MDI magnetograms (Kosovichev 2009). Panel $d$ shows a schematic flow patterns in the subsurface region beneath sunspots. The data obtained by the $\mathrm{SOHO} / \mathrm{MDI}$. 
move beyond the sunspot boundary into the surrounding region where they are observed as magnetic moving features (MMF; e.g Harvey \& Harvey 1973; Ravindra 2006).

\subsection{Subsurface dynamics of active regions}

Until recent time observations of the sunspot dynamics were limited to the solar surface, but thanks to new methods of local helioseismology we start getting new information about the development of active regions in deeper layers. The idea of the time-distance helioseismology approach (Duvall et al. 1993) is to cross-correlate the acoustic noise between different regions at the solar surface that gives measurements of the acoustic travel-times. The travel-times can be converted into sound-speed perturbations and flows of subsurface structure by using acoustic tomography (e.g., Kosovichev 1996; Kosovichev \& Duvall 1997; Lindsey \& Braun 2000; Kosovichev 2012). Figure 3a-c shows an example of development of the active region NOAA 8131 (Kosovichev 2009); the wave-speed perturbations with magnitude of about $\pm 1 \mathrm{~km} / \mathrm{s}$ resemble appearance of the filamentary magnetic structure during the magnetic flux emergence. Panel $d$ illustrates a complicated sunspot dynamics below the solar surface, where the near-surface layers (up to $4 \mathrm{Mm}$ ) are characterized by a converging flows in the relatively cold region, and diverging hotter flows are located in deeper layers below a sunspot. The high-resolution helioseismology data which became available from new advanced space instruments: HMI/SDO (Scherrer et al. 2012) and SOT/Hinode (Tsuneta et al. 2008) made it possible to detect the subsurface flow dynamics on the scales much smaller than a sunspot. For instance, the temperature increase and local diverging flows under a sunspot bridge, inhomogeneity in the velocity distribution with the sunspot structure, where downflows are more prominent, and also some indications on a supergranulation-type convective pattern below sunspots were detected (Zhao et al. 2010).

Further the progress in the helioseismology data analysis opened the possibility to detect the emergence of active regions (Ilonidis et al.2011) 1 - 2 days before the appearance on the surface. Measurements of travel-times anomalies by a specially designed technique showed the appearance of an emerging flux in the depth range of $66-57 \mathrm{Mm}$, but after passing these layers the signal disappears, probably due to strong turbulent flows near the surface, which make the emerging flux less concentrated (Cheung et al. 2007, 2008). According to these measurements a typical size of emerging region at a depth of about $60 \mathrm{Mm}$ below the surface is of the order of $30-50 \mathrm{Mm}$, and emergence speed is 0.3 - $0.6 \mathrm{~km} / \mathrm{s}$. Of course, at present time this methodology has limitations, which allow to detect only large active regions. However, it already have given some constrains for numerical modeling.

\section{Theoretical models of origin and structure of sunspots}

Magnetic flux emergence is the most visible stage of the sunspot formation process. Nevertheless, the questions on where this is flux formed, and what its are initial properties, are still open. There are two main ideas about the location of initial magnetic flux ropes: in the bottom and top layers of the convective zone, which are also identified as the regions of strong radial gradient of the rotation rate: the tachocline and subsurface shear layer. Models of the flux ropes in the tachocline clearly shows the connection of sunspot formation with the global dynamo action, and explains the orientation of active regions with respect to the equator (Joy's law), the Hale's hemispheric polarity law and others. A disadvantage of the models is that an extremely strong magnetic field (up to $100 \mathrm{kG}$ ) must be generated in the convective zone, with the energy density for exceeding the turbulent kinetic energy, representing a problem for dynamo theories. Also, it is known that 
the active regions rotate on the solar surface with a speed, which corresponds to the depth of about $25 \mathrm{Mm}$, and the tachocline rotates much slower. To resolve this discrepancy it was suggested that the flux rope that emerge from the bottom of the convective zone can disconnect with the deep layers (for references and details see a review by Parker, 2009). However, this hypothesis may be inconsistent with the fact that active regions tend to appear recurrently in particular longitudinal zones over some extended periods of time. Another hypothesis suggests that the solar magnetic field is generated in the bulk of the convective zone, but is 'shaped' into the toroidal band migrating towards the equator in the subsurface shear layer (Brandenburg 2005). This idea is indirectly supported by recent helioseismology observations of emerging magnetic flux (Ilonidis et al.2011), and also by $2 \mathrm{D}$ dynamo models, in which the magnetic field is predominantly concentrated in both bottom and top layers of the convection zone, but the sunspot 'butterfly' diagram is formed about $60 \mathrm{Mm}$ below the solar surface (Pipin \& Kosovichev 2011).

During the flux emergence, large amounts small-scale magnetic field patches of both polarities appear on the solar surface. These can be considered as an ensemble of magnetic flux bundles that can be accumulated in small areas and form sunspots, e.g. due to converging flows. However, surface observations of sunspots show mostly divergent flows, and only some weak converging flows for pores. But, because sunspots and pores have the life-time often longer than several days, the stability reasons indicated on the existence of converging flows $1-10 \mathrm{Mm}$ below the surface, which can be revealed to the supergranulation dynamics (Meyer et al. 1974). Then, magnetic bundles can be hold together by one or several surrounding vortex rings that can make the dynamics of the magnetic structure more stable (Parker 1992). The hydrodynamic interaction of the bundles due to the vortex attraction can cause their merging into single large-scale structures, like pores and sunspots. However, Parker (1979) argued that the formation of a monolithic structure (Fig. 4a) would be unstable, but a relatively loose clustering of the bundles in subsurface layers can resolve this problem, because due to the continuous vortex flow interactions they can stay together (Fig. $4 b$ ).

To explain a complex structure of the penumbra, numerous models were suggested, which were able to explain some of the observed effects and properties. The Evershed effect (Evershed 1900) was most intensively investigated where properties of the radial outflows were explained by a siphon model (Meyer \& Schmidt 1968; Thomas 1988), a model with convective rolls (Danielson 1961, Busse 1987, Hurlburt et al. 2000), and a
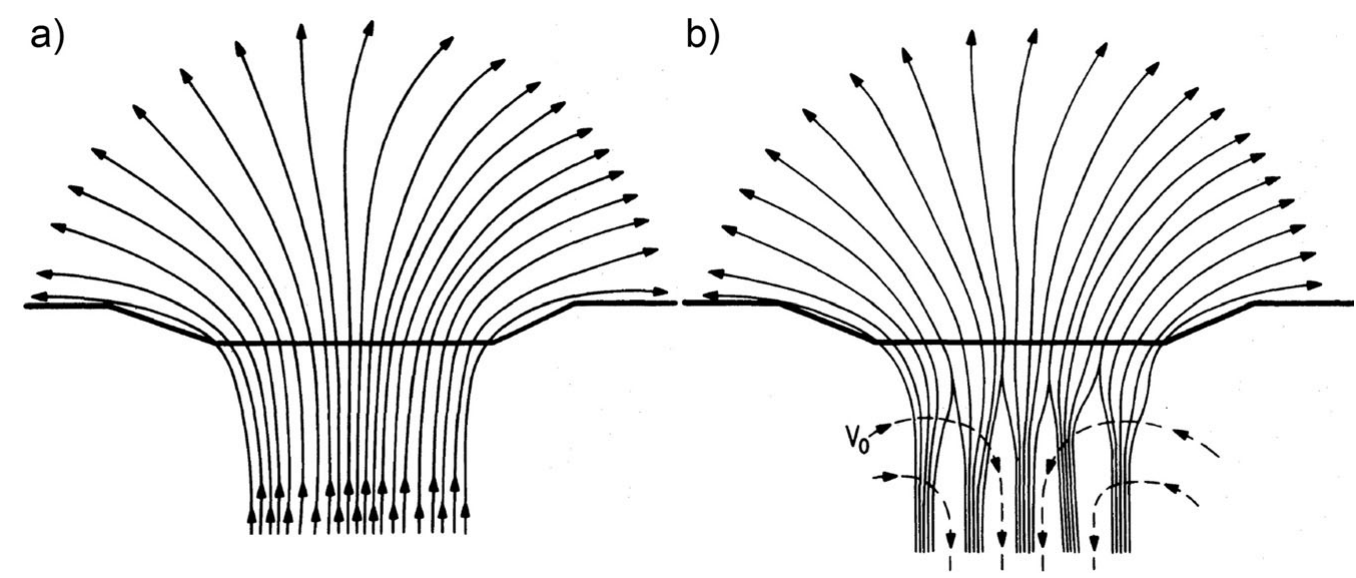

Figure 4. Monolith (panel $a$ ) and cluster (b) models of sunspot structure (Parker 1979). 
gap model (Severnyi 1965). The topological filamentary structure of penumbral magnetic field was reflected in investigations of many authors (e.g., Severnyi 1965; Solanki \& Montavon 1993, Sainz Dalda \& Bellot Rubio 2008 and others, additional references see reviews by Solanki (2003); Parker (2009)). However, our current understanding of the Evershed effect and its relationship to the filamentary structure achieved only recently by comparing high-resolution observations with realistic radiative MHD numerical simulations. It turned out that the key mechanism of the penumbra structure and flows is turbulent magnetoconvection in the strongly inclined magnetic field (Scharmer et al. 2008; Kitiashvili et al. 2009; Rempel et al. 2009).

\section{Numerical models of pores and sunspots}

Complexity of dynamics the solar MHD often makes it impossible to find analytical descriptions of observed processes without significant simplifications. Development of numerical approach to the modeling of the solar magnetoconvection approach allow us to build the physically more realistic model. For instance, one of the first numerical models of the Evershed effect, explained by a thin flux tube model (Schlichenmaier et al. 1998). Also, the pioneering work of the $2 \mathrm{D}$ overturning convection, the traveling waves in the horizontal magnetic field of a sunspot penumbra was predicted (Brownjohn et al. 1995; Hurlburt et al. 1996).

Dramatically growing of computational power make possible to have a significant progress in 'first principles' the numerical modeling (e.g., Nordlund \& Stein 2001), where background model is the standard solar model (Christensen-Dalsgaard et al. 1996), and effects of radiative transfer, magnetic effects and others are included. This approach opens new possibilities for investigation of local properties of solar magnetoconvection for particular physical conditions, including magnetic self-organization processes on different temporal and spatial scales. The simulations have been used for investigation of general properties of granulation, flow dynamics, oscillations and turbulence (e.g., Stein \& Nordlund 1989, 1998, 2001; Steiner et al. 1998; Skartlien et al. 2000; Georgobiani et al. 2000, 2003; Vögler et al. 2005; Jacoutot et al. 2008a, 2008b; Kitiashvili et al. 2011a), and also for specific local features, such as vortex tubes (e.g., Kitiashvili et al. 2010, 2011b, 2012; Steiner et al. 2010; Shelyag et al. 2011), umbral dots (Schüssler \& Vögler 2006, Bharti al. 2010), penumbra dynamics (e.g., Scharmer et al. 2008; Kitiashvili et al. 2009; Rempel 2011) and others. These small-scale features are formed naturally by magnetoconvection from uniform initial conditions. However, modeling of large-scale structures has been problematic, probably because of the relatively small computational domains restricted by the available supercomputer resources.

The first step to model a whole 'large-scale' magnetic structure by prescribing a special 'initial' conditions with a preexisting structure was done for a solar pore (Cameron et al. 2007). Later, such simulations were extended for a whole sunspot, and provided an opportunity to investigate not only local properties of the sunspot structure, but also to consider the umbra-penumbra transition, and links between the dynamics of individual features and the general surface and subsurface properties (Rempel et al. 2009, 2011a,b). However, in these simulations stability of the sunspot structure is provided by the boundary conditions fixing the magnetic field strength and geometry at the bottom and top of a shallow computational domain. When the boundary conditions are relaxed, the sunspot structure is quickly dispersed within $30 \mathrm{~min}$ for the sunspot in $8-\mathrm{Mm}$ box domain (Rempel et al. 2011) and about 24 hours for 'sunspot' without penumbra in box domain $16 \mathrm{Mm}$ deep (Rempel 2012). Thus, it is important to investigate the mechanisms of stable self-organization. 

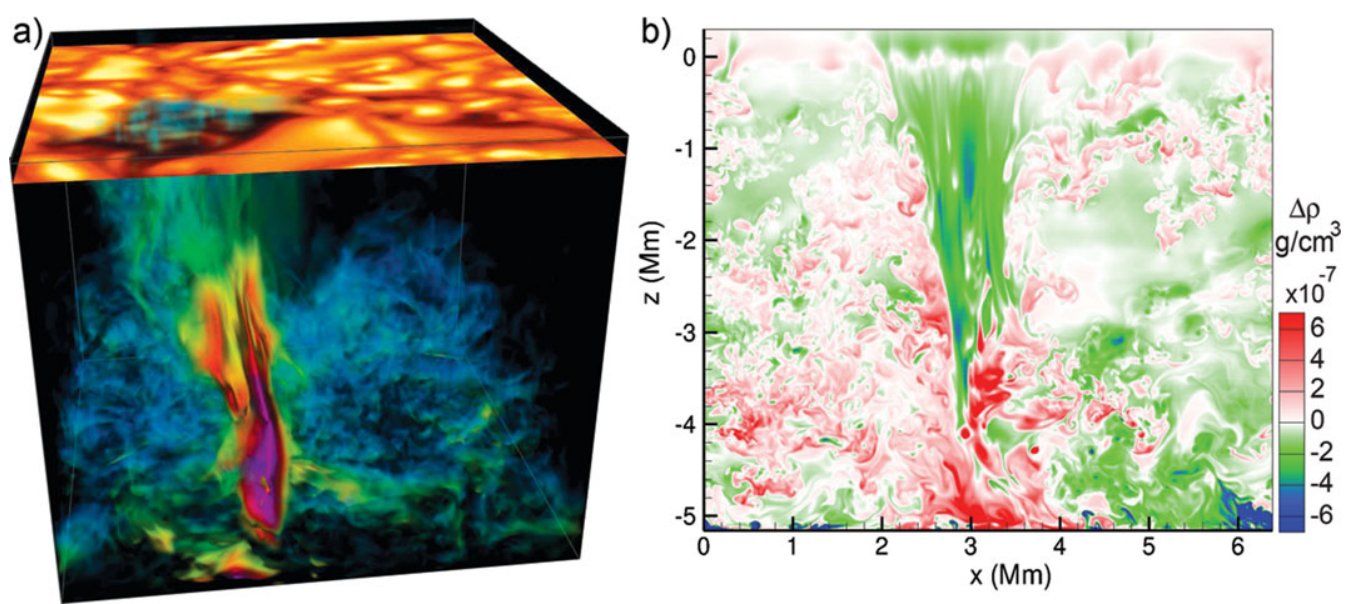

Figure 5. a) 3D rendering of spontaneously formed pore: filamentary structure of magnetic field distribution with strength ranging from $6 \mathrm{kG}$ below surface (red-magenta color) to $\sim 1.5 \mathrm{kG}$ (green) at the solar the surface. The top horizontal plane show the temperature distribution in the photosphere, displaying umbral dots and surrounding granulation. $b$ ) a vertical slice through magnetic structure, showing density variations. A thin photospheric layer of higher density (white) in the pore is associated with slowly evolving umbral dots. For details see Kitiashvili et al. (2010).

The first example of spontaneous formation of stable magnetic structures was obtained by Kitiashvili et al. (2010) in the simulations for a 5-Mm deep box domain, where after introducing an uniform $100 \mathrm{G}$ vertical magnetic field a pore-like magnetic structure was formed during about $40 \mathrm{~min}$. Analysis of these simulations showed that strong helical motions in the subsurface layers played a critical role in this process. The self-formed pore had the magnetic field of $\sim 6 \mathrm{kG}$ below surface and $\sim 1.5 \mathrm{kG}$ at the photosphere (Fig. 5), and consisted of an evolving bundles of magnetic flux tubes, coupled together by strong converging and vortical downdraft, quite similar to Parker's cluster model (Fig. 4b). On the surface, these bundles represented nonuniformly distributed umbral dots. This magnetic structure is very stable with life-time more than 8 hours, but because the boundary conditions preserved only the total magnetic flux in the domain, without prescribing any topological properties, the structure evolved with time. In this case, the structure formation is driven by local converging flows associated with vortex tubes of magnetoconvection, and represents a process of the magnetic field collapse. These simulations showed that spontaneous self-organization of initially diffuse magnetic flux may occur soon after its emergence on the surface, and that the structure formation and stability are controlled by subsurface flow. However, the process of magnetic flux emergence was not modeled in these simulations.

Recently, the simulations of flux emergence are presented by Stein (2012a), who considered for 20-Mm deep box domain and introduced a horizontal untwisted $20 \mathrm{kG}$ field feeded by upflows at the bottom boundary (in some cases the field strength was $40 \mathrm{kG}$ and $5 \mathrm{kG}$, see Stein 2012b). In these simulations, surface of magnetic structures (pores) start forming after 20 hours after the field initialization. Surprisingly, the speed of emergence was much slower than expected even for very strong initial field suppressed by downward pumping, and only relatively small structures without penumbra were formed. Nevertheless, it is important that the process of formation of these pore-like structures and their dynamics was similar to presented early by Kitiashvili et al. (2010) for the simulations with introduced weak vertical magnetic field. In context of mechanisms of magnetic field 
structurization theoretical and numerical investigation of the convective collapse due to the negative effective magnetic pressure instability (NEMPI, Kleeorin et al. 1990; Rogachevskii \& Kleeorin 2007; Brandenburg et al. 2011), explaining the tendency of the initial field to concentrate in low-pressure areas, is very important.

An efficient way to accelerate the flux emergence is to twist the initial magnetic flux rope. It was shown that the emergence speed of twisted flux ropes is higher (e.g., Murray et al. 2006; Cheung et al. 2008). Also the idea of emergence of twisted flux ropes is consistent with observed swirling motions during the emergence and sunspot formation. Simulations of emergence of a twisted semi-torus flux structure resembled formation of a bipolar active region (Cheung et al. 2010). However, the newly formed sunspots quickly lost their penumbral structure. Another example of a twisted flux rope emergence from the depth of $10 \mathrm{Mm}$ below the photosphere with an initial field strength of $50 \mathrm{kG}$ (Fang et al. 2012) showed a formation of bipolar pore-like structures, but on the scale much smaller than the active regions (order of regular granules, which have size much larger, then observed).

\section{Discussion and Conclusions}

Self-organization processes in magnetized plasma is one of the most intriguing and complicated topics in astrophysics. In particular, formation of stable magnetic structures on the solar surface, such as sunspots and pores, is a long-standing unresolved problem. Fast growing observational and numerical capabilities, accompanied by development of new modeling and data analysis methods, help us to better understand the observed features, and start developing a complete picture of formations of sunspots and pores.

In few years, we have achieved a completely new level of understanding of individual phenomena related to the dynamics of solar magnetic structures. Recent realistic radiative MHD numerical models, which can accurately represent the solar conditions, have made important steps to resolving the problem of magnetic flux emergence and formation of sunspots and pores, and gave us new directions for future investigations.

To summarize I would like to briefly overview the achievements and problems of the current numerical models. The realistic simulations of a whole sunspot structure are critically important for the understanding of the active region subsurface dynamics, and its interaction with the quiet-Sun convection. Currently, the most advances sunspot simulations by Rempel (2011a) can reproduce many observational effects of penumbra. However, the sunspot structure is maintained by the boundary conditions, which hold magnetic field of the penumbra highly inclined at the top boundary and keep the magnetic field highly concentrated at the bottom boundary. This model shows up only divergent subsurface flows around the sunspot structure which contradict to observations.

Modeling of the magnetic flux emergence give us knowledge of important dynamical links of the deep convective layers to the flux emergence observed on the surface. Two main numerical cases for the flux emergence are currently considered: emergence of untwisted horizontal magnetic field from the bottom boundary (e.g., Stein 2012a,b), and emergence of initially twisted flux ropes (e.g., Cheung et al. 2010; Martínez-Sykora et al. 2008; Fang et al. 2012). The simulations with the initially imposed untwisted horizontal field showed a spontaneous formation of pore-like structures when the magnetic flux reached the surface, but the emergence process took long time, and to accelerate this process additional upflow pumping at the bottom boundary was introduced. The reality of such magnetic flux 'feeding' is unclear. The twisted magnetic flux ropes with geometry of the semitorus or pipe-like structures able to emerge quickly keeping their relatively compact structure, which can resemble bipolar active regions on the surface. However, 
penumbral-like structure formed only for a case of a strongly twisted semitorus flux rope, and evolved very fast during the emergence, and started decay almost immediately (Cheung et al. 2010). In other cases, no signatures of the filamentary structure was presented in the twisted flux simulations. Also, as the results of Fang et al. (2012) showed that it is very important to model accurately the radiative processes because a simplified modeling of the radiative transfer can give a wrong scale of granulation (about 2 times larger than observed). This requirement puts limit on the size of simulation domains, which can be achieved in modern supercomputers. Despite the limitations the simulations show an importance of the subsurface and atmosphere turbulent dynamics during the flux emergence and formation of magnetic structures. The problem of spontaneous formation of compact self-organized stable structures from the relatively diffused emerging magnetic field is particularly interesting and important.

The simulations have provided two qualitatively similar examples of spontaneous formation of pore-like structures $\dagger$ for the cases with very different magnetic field initialization: 1) an initially weak uniform vertical magnetic field that mimics an emerging diffuse magnetic flux (Kitiashvili et al. 2010), and 2) emergence of a strong horizontal field feeded at the bottom boundary (Stein 2012). In both cases, the compact magnetic structures maintained by self-organized turbulent converging downdrafts below the surface. This means that in the subsurface flow dynamics is critically important for pore and sunspot formation.

Currently we do not have a complete model of the sunspot formation. The simulation have show an importance of detailed modeling the turbulent subsurface dynamics and radiative processes at the surface, and revealed difficulties of modeling self-formed strong horizontal fields forming filamentary structure of penumbra. This was modeled only by prescribing specific boundary conditions for inclined field. Thus, the problems of magnetic flux emergence and sunspot formation will remain is most challenging problems in astrophysics for the near future.

\section{References}

Bellot Rubio, L. R., Langhans, K., \& Schlichenmaier, R. 2005, A\&̛A, 443, L7

Brandenburg, A. 2005, ApJ, 625, 539

Brandenburg, A., Kemel, K., Kleeorin, N., Dhrubaditya M., \& Rogachevskii, I. 2011, ApJ, 740, L50

Bharti, L., Beeck, B., \& Schüssler, M. 2010, A\&A, 510, id.A12

Bray, R. J. \& Loughhead, R. E. 1964, Sunspots. London: Chapman \& Hall

Brownjohn, D. P., Hurlburt, N. E., Proctor, M. R. E., \& Weiss, N. O. 1995, J. Fluid Mech., 300, 287

Busse, F. H. 1987, The Role of Fine-Scale Magnetic Fields on the Structure of the Solar Atmosphere, ed. E.-H. Schröter, M. Vázquez \& A. A. Wyller (Cambridge: Cambridge Univ. Press), 187

Cabrera Solana, D., Bellot Rubio, L. R., Borrero, J. M., \& Del Toro Iniesta, J. C. 2008, A\&A, 477,273

Cameron, R., Schüssler, M., Vögler, A., \& Zakharov, V. 2007, A\& A, 474, 261

Cheung, M. C. M., Schüssler, M., \& Moreno-Insertis, F. 2007, A\& A, 467, 703

Cheung, M. C. M., Schüssler, M., Tarbell, T. D., \& Title, A. 2008, ApJ, 687, 1373

Cheung, M. C. M., Rempel, M., Title, A., \& Schüssler, M. 2010, ApJ, 720, 233

$\dagger$ It is important note about definition of sunspot. Historically, the sunspot is a compact structure, where umbra and penumbra regions are present. It means all simulation results, where a magnetic structure have not penumbra cannot be considered as a sunspot. Same situation for active region formation, where conglomerate of several pores without a sunspot is not an active region 
Christensen-Dalsgaard, J., Däppen, W., Ajukov, S. V., Anderson, E. R., Antia, H. M., Basu, S., Baturin, V. A., Berthomieu, G., Chaboyer, B., Chitre, S. M., Cox, A. N., Demarque, P., Donatowicz, J., Dziembowski, W. A., Gabriel, M., Gough, D. O., Guenther, D. B., Guzik, J. A., Harvey, J. W., Hill, F., Houdek, G., Iglesias, C. A., Kosovichev, A. G., Leibacher, J. W., Morel, P., Proffitt, C. R., Provost, J., Reiter, J., Rhodes Jr., E. J., Rogers, F. J., Roxburgh, I. W., Thompson, M. J. \& Ulrich, R. K. 1996, Science, 272, 1286

Danielson 1961, ApJ, 134, 289

Duvall, T. L., Jr., Jefferies, S. M., Harvey, J. W., \& Pomerantz, M. A. 1993, Nature, 362, 430

Georgobiani, D., Kosovichev, A. G., Nigam, R., Nordlund, Å., \& Stein, R. F. 2000, ApJ, 530, L139

Georgobiani, D., Stein, R. F., \& Nordlund, Å 2003, ApJ, 596, 698

Evershed, J. 1909, MNRAS, 69, 454

Fang, F., Manchester, W. I.V, Abbett, W. P., \& van der Holst, B. 2012, ApJ, 745, 37

Harvey, K. \& Harvey, J. 1973, Solar Physics, 28, 61

Hirzberger, J. 2003, A\& A, 405, 331

Hurlburt, N. E., Mettews, P. C., \& Proctor, M. R. E. 1996, ApJ, 457, 933

Ichimoto, K., Suematsu, Y., Tsuneta, S., Katsukawa, Y., Shimizu, T., Shine, R. A., Tarbell, T. D., Title, A. M., Lites, B. W., Kubo, M., \& Nagata, S. 2007, Science, 318, 1597

Ilonidis, S., Zhao, J. \& Kosovichev A. G. 2011, Science, 333, 993

Jacoutot, L., Kosovichev, A. G., Wray, A. A., \& Mansour, N. N. 2008a, ApJ, 682, 1386

Jacoutot, L., Kosovichev, A. G., Wray, A. A., \& Mansour, N. N. 2008b, ApJ, 684, L51

Keil, S. L., Balasubramaniam, K. S., Smaldone, L. A., \& Reger, B. 1999, ApJ, 510, 422

Kiepenheuer, K. O. 1953, The Sun, G.P. Kuiper (ed.), Univ. of Chicago Press, Chicago

Kitiashvili, I. N., Kosovichev, A. G., Wray, A. A., \& Mansour, N. N. 2009, ApJ, 700, L178

Kitiashvili, I. N., Kosovichev, A. G., Wray, A. A., \& Mansour, N. N. 2010, ApJ, 268, 283

Kitiashvili, I. N., Kosovichev, A. G., Mansour, N. N., \& Wray, A. A. 2011a, Solar Physics, 719, 307

Kitiashvili, I. N., Kosovichev, A. G., Mansour, N. N., \& Wray, A. A. 2011b, ApJ, 727, L50

Kitiashvili, I. N., Kosovichev, A. G., Mansour, N. N., Lele, S. K., \& Wray, A. A. 2012, Physica Scripta, 86, 018403

Kleeorin, N. I., Rogachevskii, I. V., \& Ruzmaikin, A. A. 1990, Sov. Phys. JETP, 70, 878

Kosovichev, A. G. 1996, ApJ, 461, L55

Kosovichev, A. G. 2009, Space Sci. Rev., 144, 175

Kosovichev, A. G. 2012, Solar Physics, 279, 323

Kosovichev, A. G. \& Duvall, T. L., Jr. 1997, Proc. of a workshop 'SCORe'96: Solar Convection and Oscillations and their Relationship', Aarhus, Denmark, May 27 - 31, 1996, Eds.: F.P. Pijpers, J. Christensen-Dalsgaard, and C.S. Rosenthal, Kluwer Academic Publishers (Astrophysics and Space Science Library Vol. 225), 241

Langhans, K., Scharmer, G. B., Kiselman, D., L̈̈dahl, M. G., \& Berger T. E. 2005, A\&A, 436, 1087

Lindsey, C. \& Braun, D. C. 2000, Science, 287, 1799

Loughhead, R. E. \& Bray, R. J. 1961, Aust. J. Phys., 14, 347

Martínez Pillet, V. 2000, AESA, 3618, 734

Márquez, I., Sánchez Almeida, J., \& Bonet, J. A. 2006, ApJ, 638, 553

Martínez-Sykora, J., Hansteen, V., \& Carlsson, M. 2008, ApJ, 679, 871

McIntosh, P. S. 1990, Solar Physics, 125, 251

Meyer, F. \& Schmidt, H. U. 1968, Mitt. Astron. Ges., 25, 194

Meyer, F., Schmidt, H. U., Wilson, P. R., \& Weiss, N. O. 1974, MNRAS, 169, 35

Murray, M. J., Hood, A. W., Moreno-Insertis, F., Galsgaard, K., \& Archontis, V. 2006, A\&A, 460,909

Nordlund, Å. \& Stein, R. F. 2001, ApJ, 546, 576.

Ortiz, A., Bellot Rubio, L. R., \& Rouppe van der Voort, L. 2010, ApJ, 713, 1282

Parker, E. N. 1979, ApJ, 230, 905

Parker, E. N. 1992, ApJ, 390, 290

Parker, E. N. 2009, Space Sci. Rev., 144, 15 
Ravindra, B. 2006, Solar Physics, 237, 297

Rempel, M., Schüssler, M., Cameron, R. H., \& Knölker 2009, Science, 325, 171

Rempel, M. 2011a, ApJ, 729, 5

Rempel, M. 2011b, ApJ, 740, 15

Rempel, M., Cheung, M., Birch, A. C., \& Braun, D. C. 2011, American Geophysical Union, Fall Meeting 2011, abstract SH52B-02

Rempel, M. 2012, ApJ, 750, 62

Rezaei, R., Bello González, N., \& Schlichenmaier, R. 2012, A\&AA, 537, A19

Rimmele, T. R. 2004, ApJ, 604, 906

Rimmele, T. R. 2008, ApJ, 672, 684

Rimmele, T. \& Marino, J. 2006, ApJ, 646, 593

Roudier, Th., Bonet, J. A., \& Sobotka, M. 2002, A\&SA, 395, 249

Rouppe van der Voort, L. H. M. 2003, AESA, 397, 757

Pipin, V. V. \& Kosovichev, A. G. 2011, The Asymmetry of Sunspot Cycles and Waldmeier Relations as a Result of Nonlinear Surface-shear Shaped Dynamo ApJ, 741, 1

Rogachevskii, I. \& Kleeorin, N. 2007, Phys. Rev. E, 76, 056307

Sainz Dalda, A., Vargas, J. A., \& Sobotka, M. 2002, A\&GA, 395, 249

Sainz Dalda, A. \& Bellot Rubio, L. R. 2008, A\& A, 481, L21

Sánchez Almeida, J. 2005, ApJ, 622, 1292

Sankarasubramanian, K. \& Rimmele, T. 2003, ApJ, 598, 689

Schlichenmaier, R., Jahn, K., \& Schmidt, H. U. 2010, ApJ, 493, L121

Schlichenmaier, R., Rezaei, R., Bello González, N., \& Waldmann, T. A. 2010, A\& A, 512, L1

Scharmer, G. B., Gudiksen, B. V., Kiselman, D., Löfdahl, M. G., \& Rouppe van der Voort, L. H. M. 2002, Nature, 420,151

Severnyi, A. B. 1959, Soviet Astronomy, 3, 214

Severnyi, A. B. 1965, Soviet Astronomy, 9, 171

Skartlien, R., Stein, R. F., \& Nordlund, A. 2000, ApJ, 541, 468

Scharmer, G. B., Nordlund, Å., \& Heinemann, T. 2008, ApJ, 677, L149

Scherrer, P. H., Schou, J., Bush, R. I., Kosovichev, A. G., Bogart, R. S., Hoeksema, J. T., Liu, Y., Duvall, T. L., Zhao, J., Title, A. M., Schrijver, C. J., Tarbell, T. D., \& Tomczyk, S. 2012, Solar Physics, 275, 207

Schüssler, M. \& Vögler, A. 2006, ApJ, 641, L73

Shine, R. A., Title, A. M., Tarbell, T. D., Smith, K., Frank, Z. A., \& Scharmer, G. 1994, ApJ, 430,413

Shelyag, S., Keys, P., Mathioudakis, M., \& Keenan, F. P. 2011, A\&AA, 526, id.A5.

Sobotka, M., Vázquez, M., Bonet, J. A., Hanslmeier, A., \& Hirzberger J. 1999, ApJ, 511, 436

Sobotka, M. 2003, Astron. Nachr., 324, 369

Sobotka, M. \& Puschmann, K. G. 2009, A\& A, 504, 575

Socas-Navarro, H., Pillet, V. M., Sobotka, M., \& Vázquez, M. 2004, ApJ, 614, 448

Solanki, S. K. \& Montavon, C. A. P. 1993, A\& A, 275, 283

Solanki, S. K. 2003, Astron. Astrophys.Rev., 11, 153

Stenflo, J. O. \& Kosovichev, A. G. 2012, ApJ, 745, 129

Stein, R. F. \& Nordlund, Å. 1989, ApJ, 342, L95

Stein, R. F. \& Nordlund, Å. 1998, ApJ, 499, 914

Stein, R. F. \& Nordlund, A. 2001, ApJ, 546, 585

Stein, R. F., Lagerfjärd, A., Nordlund, Å., \&. Georgobiani, D. 2012a, ASP Conf. Series, 455, 133

Stein, R. F. 2012b, Living Rev. in Solar Phys., 9, 4

Steiner, O., Grossmann-Doerth, U., Knoelker, M., \& Schuessler, M. 1998, ApJ, 495, 468

Steiner, O., Franz, M., Bello González, N., Nutto, Ch., Rezaei, R., Martínez Pillet, V., Bonet Navarro, J. A., del Toro Iniesta, J. C., Domingo, V., Solanki, S. K., Knölker, M., Schmidt, W., Barthol, P., \& Gandorfer, A. 2010, ApJ, 723, L180

Strous, L. H. \& Zwaan, C. 1999, ApJ, 527, 435

Sutterlin, P., Schröterm E. H. \& Muglach, K. 1996, Solar Physics, 164, 311

Thomas, J. H. 1988, ApJ, 333, 407 
Tsuneta, S., Ichimoto, K., Katsukawa, Y., Nagata, S., Otsubo, M., Shimizu, T., Suematsu, Y., Nakagiri, M., Noguchi, M., Tarbell, T., Title, A., Shine, R., Rosenberg, W., Hoffmann, C., Jurcevich, B., Kushner, G., Levay, M., Lites, B., Elmore, D., Matsushita, T., Kawaguchi, N., Saito, H., Mikami, I., Hill, L. D., \& Owens, J. K. 2008, Solar Physics, 249, 167

Vögler, A., Shelyag, S., Schüssler, M., Cattaneo, F., Emonet, T., \& Linde, T. 2005, A\&A, 429, 335

Waldmeier, M. 1947, Publ. Zurich Obs., 9, 311

Wang, H. \& Zirin, H. 1992, Solar Physics, 140, 41

Watanabe, H., Kitai, R., \& Ichimoto, K. 2009, ApJ, 702, 1048

Westendorp Plaza, C., del Toro Inesta, J. C., Ruiz Cobo, B., Martenez Pillet, V., Lites, B. W., \& Skumanich, A. 1997, Nature, 389, 47

Zhao, J., Kosovichev, A. G., \& Sekii, T. 2010, ApJ, 708, 304

Zirin, H. 1972, Solar Physics, 22, 34

Zirin, H. 1974, IAUSymp. 56, R.G. Athay (ed.), 161

Zirin, H. \& Wang, H. 1992, ApJ, 385, L27

Zuccarello, F., Romano, P., Guglielmino, S. L., Centrone, M., Criscuoli, S., Ermolli, I., Berrilli, F., \& Del Moro, D. 2009, A\& A, 500, L5

Zwaan, C. 1985, Solar Physics, 100, 397 